\title{
Proposed Treatment Protocol for Frostbite: A Retrospective Analysis of 17 Cases Based on a 3-Year Single-Institution Experience
}

\author{
Eun-Kyung Woo, Jong Wook Lee, Gi-Yeun Hur, Jang-Hyu Koh, Dong-Kook Seo, Jai-Ku Choi, \\ Young-Chul Jang \\ Department of Plastic and Reconstructive Surgery, Hallym University Hangang Sacred Heart Hospital, Hallym University College of \\ Medicine, Seoul, Korea
}

Background This paper discusses the treatment protocol for patients with frostbite.

Methods We performed a retrospective analysis of a series of 17 patients with second- and higher-degree frostbite who had been treated at our medical institution between 2010 and 2012.

Results Our clinical series of patients $(n=17)$ included 13 men and four women, whose mean age was $42.4 \pm 11.6$ years (range, 22-67 years). The sites of injury include the foot in six patients (35.3\%), the hand in six patients (35.3\%) and the facial region in five patients (29.4\%). Seven patients with second-degree frostbite were completely cured with only conservative treatment during a mean period of $12.7 \pm 3.3$ days (range, 8-16 days). Of the five patients with third-degree frostbite, two underwent skin grafting following debridement, and the remaining three achieved a complete cure with conservative treatment during a mean period of $35 \pm 4.3$ days (range, 29-39 days). Five patients with fourth-degree frostbite were treated with surgical procedures including amputation.

Conclusions With the appropriate conservative management in the early stage of onset, surgeons should decide on surgery after waiting for a sufficient period of time until the demarcation of the wound. Continuous management of patients is also needed to achieve functional recovery after a complete cure has been achieved. This should also be accompanied by patient education for the avoidance of re-exposure to cold environments.

Keywords Frostbite / Therapy / Operative surgical procedure

\author{
Correspondence: Jong Wook Lee \\ Department of Plastic and \\ Reconstructive Surgery, Hallym \\ University Hangang Sacred Heart \\ Hospital, Hallym University College \\ of Medicine, 12 Beodeunaru-ro 7-gil, \\ Yeongdeungpo-gu, Seoul 150-719, \\ Korea \\ Tel: +82-2-2639-5704 \\ Fax: +82-2-2676-2431 \\ E-mail:jwlps@hallym.or.kr
}

No potential conflict of interest relevant to this article was reported.

\section{INTRODUCTION}

Frostbite is defined as tissue damage occurring when the human body is exposed to temperatures below the freezing point $\left(0^{\circ} \mathrm{C}\right)$. It is a local cold injury that commonly occurs in the extremities or facial organs such as the ear or nose. Its high prevalence in soldiers or professional mountaineers has been well documented. Recent studies have shown, however, that it has also increasingly occurred in a general group of people who frequently climb mountains and are thus more often exposed to extremely cold

Copyright (C) 2013 The Korean Society of Plastic and Reconstructive Surgeons

This is an Open Access article distributed under the terms of the Creative Commons Attribution Non-Commercial License (http://creativecommons.org/

licenses/by-nc/3.0/) which permits unrestricted non-commercial use, distribution, and reproduction in any medium, provided the original work is properly cited 


\begin{tabular}{|l}
\hline Table 1. Classification of cold injury \\
\hline Frostnip \\
Skin becomes white and loses sensation. On rewarming becomes hyperemic \\
and paresthetic. \\
Recovers completely. Paresthesia persists for some weeks. \\
Superficial frostbite \\
1st degree \\
- Partial skin freezing \\
- Erythema, edema, and hyperemia \\
- No blisters or necrosis \\
- Occasional skin desquamation (5-10 days later) \\
2nd degree \\
- Full-thickness skin freezing \\
- Erythema, substantial edema \\
- Vesicles with clear fluid \\
- Blisters, desquamation and black eschar (gangrene) formed \\
Deep frostbite \\
3rd degree \\
- Full-thickness skin \& subcutaneous freezing \\
- Violaceous/hemorrhagic blisters \\
- Skin necrosis \\
- Blue-gray discoloration \\
4th degree \\
- Full-thickness skin, subcutaneous tissue, muscle, tendon, and bone freezing \\
- Little edema \\
- Initially mottled, deep red, or cyanotic \\
- Eventually dry, black, and mummified \\
\hline From Imray C, et al. Postgrad Med J 2009;85:481-8, with permission from British \\
Medical Journal [1]. \\
\hline
\end{tabular}

weather $[1,2]$.

Like burn injuries, frostbite is also classified based on its depth [3]. That is, as shown in Table 1, it is classified into frostnip, superficial frostbite, and deep frostbite [1,4]. In addition, its pathogenesis is based on direct cellular injury and local ischemia. Therefore, it shows a different clinical course from a burn injury $[2,5]$. Furthermore, there is also a difference in the treatment protocol between frostbite and a burn injury. In other words, unlike burn injuries, there is a tendency for healing and worsening of wounds to be delayed in cases of frostbite. Surgeons should therefore decide on surgery after waiting for a sufficient period of time. In addition, even after a complete cure has been achieved, early rehabilitation is essential for functional recovery. According to a review of the English literature, it is also recommended that surgeons wait for 3 to 8 weeks before surgery $[3,6]$. To date, however, there have been no systematic reports about the treatment protocol for patients with frostbite in Korea.

Given the above background, we performed a retrospective analysis of a series of 17 patients based on our 3-year singleinstitution experience.

\section{METHODS}

\section{Study patients}

In the current study, we performed a retrospective analysis of the medical records for the 17 patients with second- and higherdegree frostbite who were admitted to the Department of Plastic and Reconstructive Surgery of our medical institution during a period ranging from 2010 to 2012. Thus, we evaluated the age, sex, cause, site, and depth of frostbite, the month of onset of injury, predisposing factors, indications of surgery and the length of the treatment period.

\section{Treatment methods}

At our medical institution, re-warming was performed from the early stage of hospitalization. In addition, patients were prescribed an oral non-steroidal anti-inflammatory drug (NSAID) (talniflumate, $370 \mathrm{mg}$ every 12 hours) for its analgesic and antiinflammatory effects $[7,8]$. Moreover, patients were also given an intravenous injection of prostaglandin E1 (PGE1) (alprostadil, $5 \mu \mathrm{g} /$ day), which is known as a vasodilator that increases tissue survival, for 24 hours [9]. With the administration of prophylactic antibiotics, we performed dressing of the affected sites for the purposes of protecting them and promoting wound healing. After waiting for the longest time possible until the necrotized tissue became clearly visible, we performed the surgical procedure $[1,3]$.

\section{RESULTS}

\section{Demographic and clinical characteristics}

The demographic and clinical characteristics of the patients are presented in Table 2. Our clinical series of patients $(n=17)$ was composed of 13 men (76.5\%) and four women (23.5\%) whose mean age was $42.4 \pm 11.6$ years (range, $22-67$ years).

The sites of injury included the foot in six patients (35.3\%), the hand in six patients (35.3\%), and the facial region in five patients (29.4\%). As for the causes of injury, 11 patients (64.7\%) sustained frostbite while climbing a mountain, three patients (17.6\%) did so due to a loss of consciousness while sleeping outdoors, two patients (11.8\%) did while walking on the cold ground barefoot, and one patient (5.9\%) did so while being trained in the military (Table 3).

By the month of onset of injury, there were 12 patients who sustained a frostbite injury in January, which was the highest prevalence, four patients who sustained the frostbite in February, and one patient who sustained the frostbite injury in December. Of note, the number of patients who sustained the frostbite injury during climbing was the greatest. 
Table 2. Demographic and clinical characteristics

\begin{tabular}{|c|c|c|c|c|c|c|c|}
\hline Case no. & Sex/Age & $\begin{array}{l}\text { Site of } \\
\text { injury }\end{array}$ & Cause of injury & Degree & Operation & $\begin{array}{c}\text { Treatment } \\
\text { period (day) }\end{array}$ & Sequelae \\
\hline 1 & $\mathrm{M} / 40$ & Face & Climbing a mountain & 2 & - & 12 & - \\
\hline 2 & $M / 48$ & Face & Climbing a mountain & 2 & - & 14 & - \\
\hline 3 & $\mathrm{M} / 57$ & Foot & Walking on barefoot & 2 & - & 9 & Cold intolerance \\
\hline 4 & $\mathrm{M} / 51$ & Face & Climbing a mountain & 2 & - & 12 & - \\
\hline 5 & $M / 44$ & Face & Climbing a mountain & 2 & - & 8 & - \\
\hline 6 & $M / 23$ & Foot & Losing self-consciousness & 2 & - & 18 & Cold intolerance \\
\hline 7 & $F / 28$ & Foot & Walking barefoot & 2 & - & 18 & Cold intolerance \\
\hline 8 & $\mathrm{M} / 43$ & Foot & Losing self-consciousness & 3 & - & 39 & Cold intolerance, numbness \\
\hline 9 & $\mathrm{M} / 50$ & Face & Climbing a mountain & 3 & - & 29 & Cold intolerance \\
\hline 10 & $\mathrm{M} / 40$ & Hand & Climbing a mountain & 3 & - & 37 & Cold intolerance, numbness \\
\hline 11 & $F / 31$ & Hand & Climbing a mountain & 3 & + & 22 & Cold intolerance, numbness \\
\hline 12 & $F / 43$ & Hand & Climbing a mountain & 3 & + & 62 & Pain, cold intolerance, numbness \\
\hline 13 & $M / 22$ & Foot & Undergoing military training & 4 & + & 50 & Pain, cold intolerance, numbness \\
\hline 14 & $M / 54$ & Hand & Climbing a mountain & 4 & + & 62 & Pain, cold intolerance, numbness \\
\hline 15 & $\mathrm{M} / 67$ & Hand & Climbing a mountain & 4 & + & 59 & Pain, cold intolerance, numbness \\
\hline 16 & $F / 41$ & Hand & Climbing a mountain & 4 & + & 60 & Pain, cold intolerance, numbness \\
\hline 17 & $\mathrm{M} / 40$ & Foot & Losing self-consciousness & 4 & + & 68 & - (below knee amputation) \\
\hline
\end{tabular}

\section{Table 3. Cases undergoing surgery}

\begin{tabular}{|ccccccc|}
\hline $\begin{array}{c}\text { Case } \\
\text { no. }\end{array}$ & Sex/Age & $\begin{array}{c}\text { Sites of } \\
\text { injury }\end{array}$ & Degree & $\begin{array}{c}\text { 1st op- } \\
\text { eration } \\
\text { (PFD) }\end{array}$ & $\begin{array}{c}\text { Re-op- } \\
\text { eration }\end{array}$ & $\begin{array}{c}\text { Treat- } \\
\text { ment } \\
\text { period }\end{array}$ \\
\hline 11 & $\mathrm{~F} / 31$ & Hand & 3 & 12 & - & 22 \\
12 & $\mathrm{~F} / 43$ & Hand & 3 & 24 & - & 62 \\
13 & $\mathrm{M} / 22$ & Foot & 4 & 37 & - & 50 \\
14 & $\mathrm{M} / 54$ & Hand & 4 & 16 & - & 62 \\
15 & $\mathrm{M} / 67$ & Hand & 4 & 26 & + & 59 \\
16 & $\mathrm{~F} / 41$ & Hand & 4 & 17 & + & 60 \\
17 & $\mathrm{M} / 40$ & Foot & 4 & 7 & + & 68 \\
\hline
\end{tabular}

PFD, post-frostbite day.

In our clinical series of patients, predisposing factors included alcohol or drug abuse and psychiatric problems. However, there were no patients who sustained the frostbite injury during climbing or military training in whom the above predisposing factors were involved. Of the two patients who sustained the frostbite injury while walking barefoot, one had auditory hallucinations due to schizophrenia.

There were seven patients with second-degree frostbite, all of whom were completely cured with only conservative treatment during a mean period of $12.7 \pm 3.3$ days (range, 8 - 16 days). There were five patients with third-degree frostbite. Of these, two patients underwent skin grafting following debridement, and the remaining three achieved a complete cure with conservative treatment. The conservative treatment lasted for a mean period of $35 \pm 4.3$ days (range, 29-39 days). There were five patients with fourth-degree frostbite, all of whom were treated with surgical procedures, including amputation. Following the amputation, one patient underwent a groin flap, one an abdominal flap, and

\section{Fig. 1. Second-degree frostbite}

(A) A 57-year-old man with second-degree frostbite to both feet presented with erythema and vesicle formation at the time of the onset of injury. (B) The patient achieved re-epithelialization on day 9.
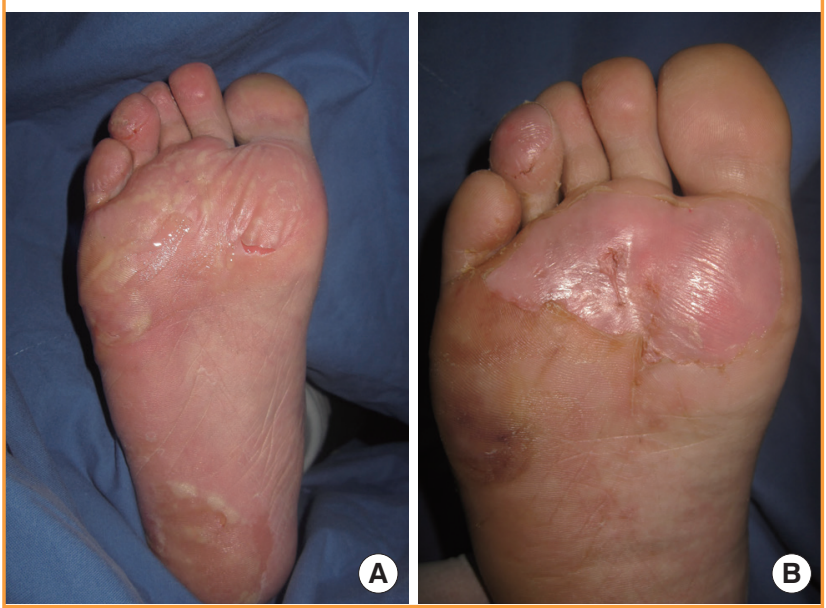

the remaining three a local flap. Surgery was performed for a total of seven patients. In six of those patients, excluding only patient 17 , however, the operation was performed within a mean period of 22.8 \pm 7.9 days (range, 12-37 days) following the onset of injury. In patient 17 , it was performed seven days after the onset of injury. Moreover, a mean period of 51.5 \pm 13.7 days (range, 22-60 days) elapsed until a complete recovery was achieved.

\section{Illustrative cases}

Case 1 (patient 3)

A 57-year-old man sustained second-degree frostbite to both feet while walking outdoors barefoot. The patient presented 


\section{Fig. 2. Third-degree frostbite}

(A) A 43-year-old woman with third-degree frostbite of both hands presented with a blue-gray discoloration at the time of the onset of injury. (B) The patient exhibited full-thickness skin necrosis on day 23. (C) The patient underwent debridement and a split-thickness skin graft on day 24. (D) The patient achieved a complete cure by postoperative week 5.
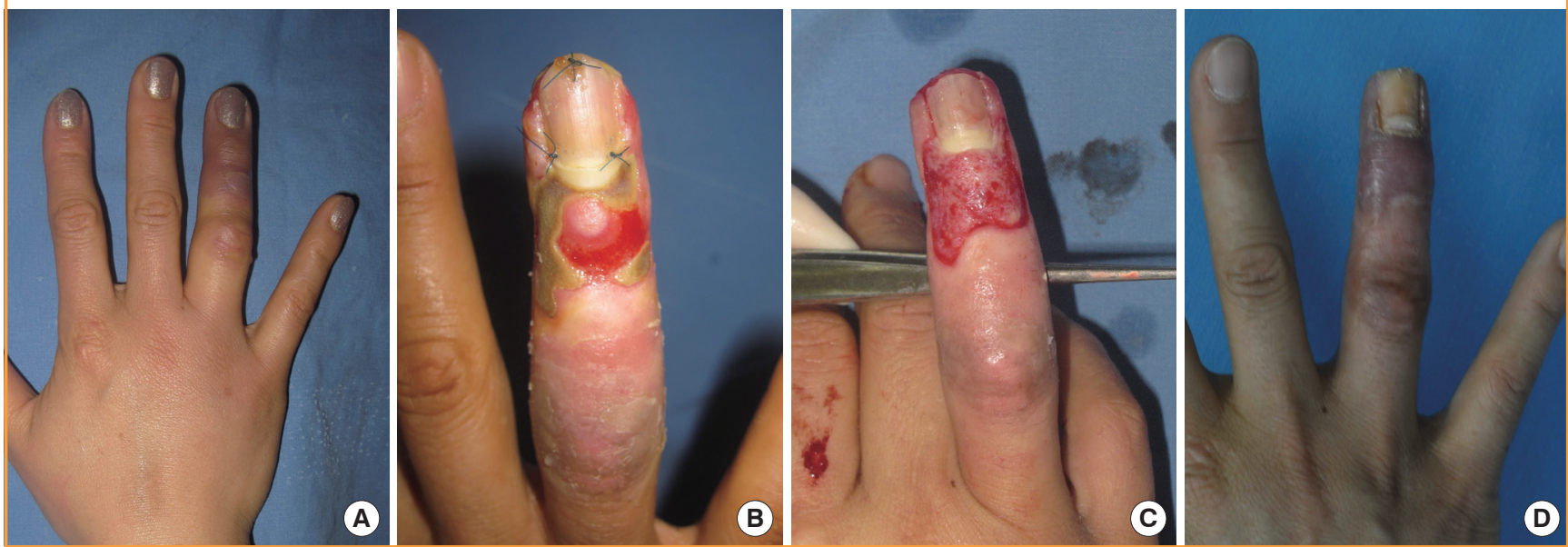

\section{Fig. 3. Fourth-degree frostbite}

(A) A 67-year-old man with third- and fourth-degree frostbite of both hands presented with a blue-gray discoloration seen at the time of the onset of injury. $(B, C)$ The patient presented with dry, black mummification on day 18 , for which we performed an amputation and reconstruction with a groin flap and local flap on day 21. (D) The patient underwent flap detachment on 16 days after the first operation.
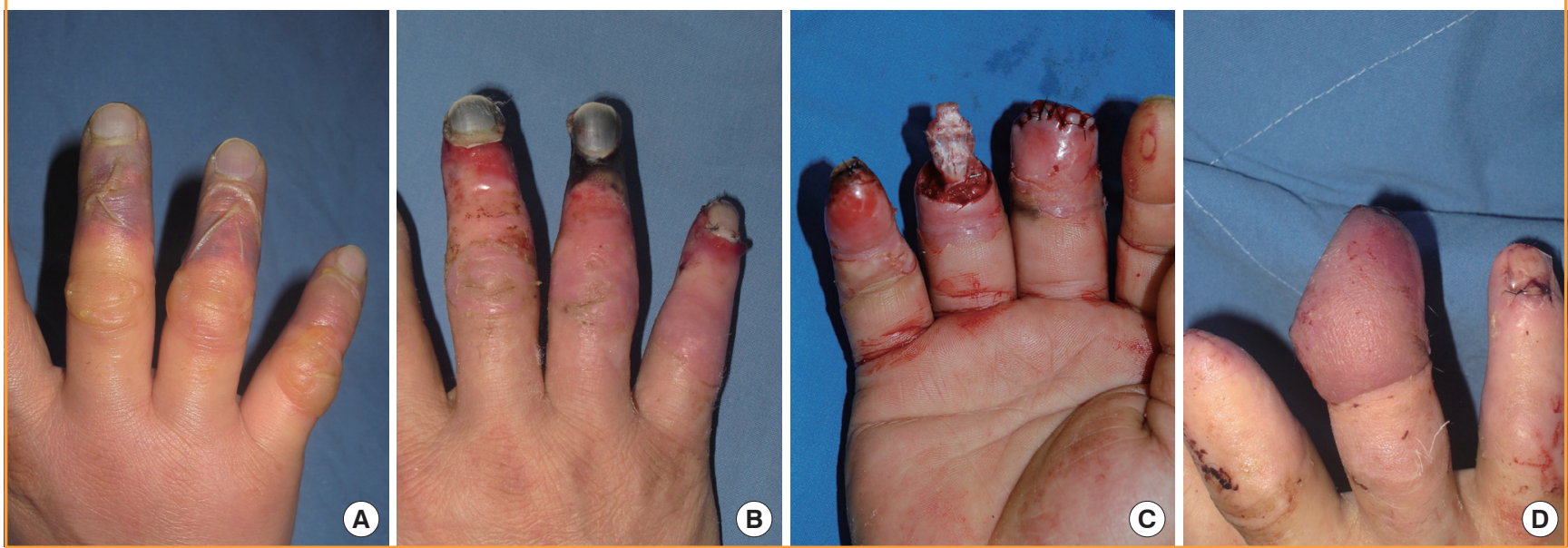

with erythema and vesicle formation at the time of the onset of injury. In addition, the patient achieved re-epithelialization by day 9. The patient was completely cured without any sequelae. Furthermore, the patient presented no gait disturbance or sensory impairment (Fig. 1).

\section{Case 2 (patient 12)}

A 43-year-old woman sustained third-degree frostbite to both hands while climbing a mountain. The patient presented with a blue-gray discoloration seen at the time of the onset of injury. In addition, the patient exhibited full-thickness skin necrosis on day 23. Therefore, the patient underwent debridement and a split-thickness skin graft on day 24 . The patient achieved a com-

plete cure on postoperative week 5 . In this patient, however, the treatment period was relatively longer; the patient received the treatment until up to postoperative week 3 (at day 56 following the onset of injury). However, the patient complained of limited joint mobility due to swelling of the hand and joint stiffness. These symptoms were improved through continuous finger movement and rehabilitation therapy. However, the patient had persistent sensory impairment (Fig. 2).

\section{Case 3 (patient 15)}

A 67-year-old man sustained third- and fourth-degree frostbite to both hands while climbing a mountain. The patient presented with a blue-gray discoloration seen at the time of the onset 


\section{Fig. 4. Fourth-degree frostbite}

(A) The patient had cyanotic lesions where the vesicles were detached at the time of the outpatient visit on day 7. (B) The patient underwent cadaveric allograft following early escharectomy in another clinical department of our medical institution on day 7. (C) The patient underwent amputation due to a progression of dry, black mummification at 1 month. (D) Below knee amputation was performed.
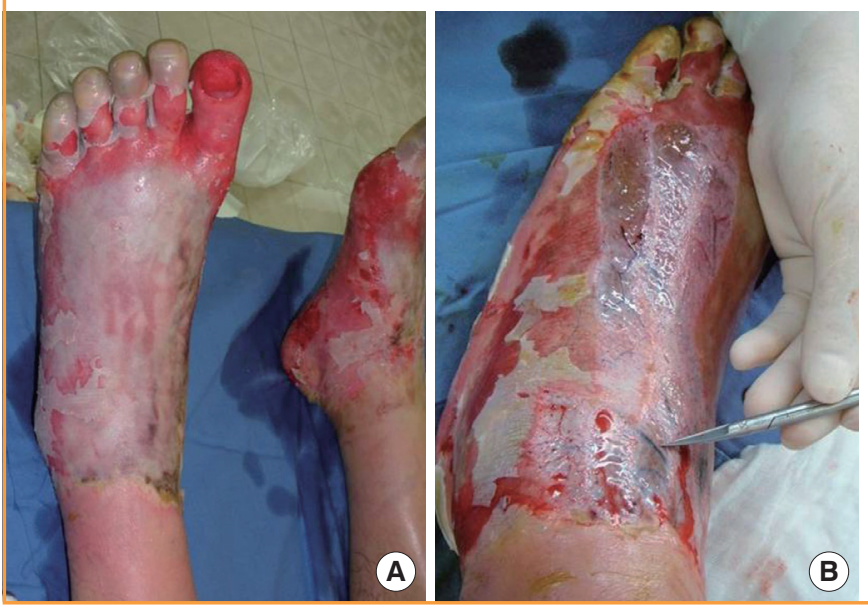

of injury. Dry, black mummification had appeared by day 18 . The patient underwent amputation and reconstruction with a groin flap and local flap on day 21. The patient underwent flap detachment 16 days after the first operation. As seen in patient 12 , despite a longer treatment period, the patient also achieved a complete cure but complained of symptoms such as limited joint mobility and sensory impairment. The limited joint mobility was greatly improved by exercise. However, the sensory impairment persisted (Fig. 3).

\section{Case 4 (patient 17)}

A 40-year-old man was referred to us after undergoing early escharectomy at seven days after the onset of injury in another clinical department. Due to the progression of necrosis in both feet over time, the patient underwent below-knee amputation at 34 days following the onset of injury. The patient was currently using a prosthetic foot (Fig. 4).

\section{DISCUSSION}

To summarize, our study showed the following results depending on the degree of frostbite injury: patients with second-degree frostbite achieved a recovery with conservative treatment alone, thus showing a favorable clinical course (Fig. 1). Some patients with third-degree frostbite were in need of surgical treatment. Patients with fourth-degree frostbite needed amputation because of injuries to such structures as the muscle, tendon, and bone.

In the early stage of the onset of injury, it is difficult to determine whether current lesions would remain as second-degree frostbite or progress to third- or higher-degree cases. Surgeons
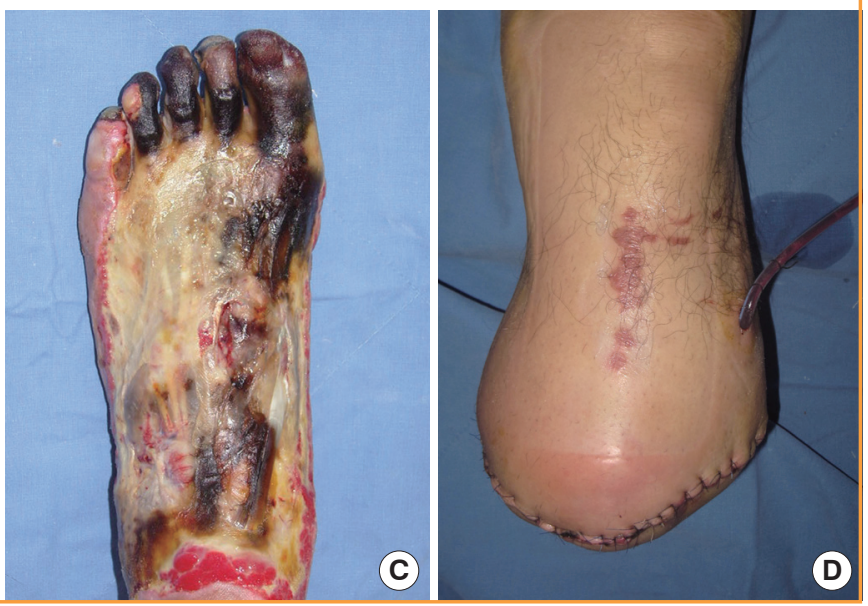

should, therefore meticulously monitor the course of the wound healing process. As illustrated in Figs. 2, 3, there is no great discrepancy in the clinical features at the time of the onset of frostbite. With the time elapsed since the clinical onset, however, they showed a difference in the depth of frostbite. Moreover, it usually takes longer for the depth of frostbite to become noticeable as compared with burns. Therefore, surgeons cannot decide on surgery without waiting for a sufficient period of time. They should also wait for more than three weeks to decide on whether and when to perform surgery. With regard to the management of frostbite injury, Cauchy et al. [10] reported that the classification of frostbite should be made based on a retrospective diagnosis, given that the final tissue loss or prognosis could not be predicted in the early stage of the onset of injury. These authors also recommended that clinicians wait for 3 to 6 weeks to determine whether patients are indicated for amputation. According to the treatment guidelines for patients with minor frostbite, proposed in the UK in 2010, it is also recommended that surgical treatment be delayed for as long as possible with conservative management [6]. In cases of major frostbite, thrombolysis can be attempted within 24 hours of the onset of injury. After 24 hours, however, surgery should be delayed for as long as possible, with imaging studies such as bone scanning, until a demarcation is achieved. In addition, it has also been suggested that early surgical debridement is contraindicated for most patients. This is not only because it takes more than several weeks for non-viable tissues to achieve a definitive demarcation but also because there is a possibility that a recovery might be achieved from the tissue injuries that were initially thought to be irreversible. Even when surgery is indicated, it is recommended that it be delayed until 6-8 weeks following the onset of the 
injury because there is a possibility that surgical trauma might interfere with the wound healing in the proximal tissue $[6,11]$. Consistent with these reports, the wound was aggravated after early escharectomy in one of our clinical cases (Fig. 4). Presumably, this might be not only because reversible tissue was removed during the melting of the frozen tissue but also because new tissue injury occurred during the surgery.

There is a consensus on the following treatment protocolsin the literature: 1) The body's core temperature should be raised, and the affected sites should be rewarmed at a temperature of $40^{\circ} \mathrm{C}$ to $42^{\circ} \mathrm{C}$. 2) Intravascular thrombosis should be prevented with the use of antithromboxane agents (e.g., ibuprofen and PEG1) and topical aloe vera gel. If already developed, microvascular thrombosis should be managed, depending on its severity, for which surgeons should use thrombolytic agents (e.g., tissue plasminogen activators) via an intravenous or intraarterial route. 3) The affected sites should be conservatively managed, if possible; no trauma should be caused, and a good wound healing environment should be created. 4) Any tissue defects should be reconstructed. 5) Sequelae of frostbite injury include pain, cold intolerance, and epiphyseal deformity (in adolescents), all of which should also be managed. 6) Patients with frostbite injury should remain under long-term follow-up during a period of 6-12 months. They should be evaluated for the possible occurrence of cartilaginous injury or bone resorption.

Regarding the predisposing factors for frostbite, Valnicek et al. [12] conducted a retrospective analysis of 125 patients based on a 12-year multi-center experience and reported that the predisposing factors include alcohol use (46\%), psychiatric problems (17\%), trauma due to a vehicle (19\%), failure of a vehicle (15\%), and drug abuse (4\%). In our clinical series of patients, the predisposing factors included alcohol or drug abuse and psychiatric problems, but there were no patients who sustained a frostbite injury during climbing or military training in whom the above predisposing factors were involved. Of the two patients who sustained a frostbite injury while walking barefoot, one had auditory hallucinations due to schizophrenia.

At our medical institution, we performed surgical procedures for our patients when they achieved a demarcation of the dead tissue on clinical findings. However, there were many cases in which the degree of tissue injury could not be confirmed on gross examination. In addition, there were also many cases in which the viable tissue could not be intraoperatively differentiated from the necrotized tissue. Furthermore, it was also frequently observed that wound healing was delayed even following the operation. This might have originated from hemodynamic derangement due to an ischemic injury to the adjacent tissue [5]. To improve these findings and to determine the optimal timing of surgery, imaging studies would be helpful. In association with this, it has been reported that technetium-99 scintigraphy and magnetic resonance angiography are particularly useful $[13,14]$.

Unlike other types of injury, the edema or joint stiffness at the sites of frostbite remain severe for longer periods of time, even after wound healing. This might occur as a result of an ischemic injury to the adjacent tissue. We therefore propose that surgeons advise patients with frostbite to wear garments in the early stage after a complete cure has been achieved, which is essential for reducing edema and preventing joint stiffness by early joint movement. In this way, a prompt recovery of the joint functions should be achieved. In addition, surgeons should also consider the possibility that long-term sequelae might occur even after a complete cure has been achieved. Most patients with frostbite complain of chronic pain, which might originate from vasomotor dysfunction. In addition, it has also been reported that many patients also complain of cold hypersensitivity, a numbness of the fingers, and reduced touch sensation, all of which might be due to thermophysiological changes in those with an increased tendency to vasospasm. Thus, a single episode of frostbite can result in cold sensitization (inability to tolerate cold temperatures in the affected sites) and predispose the site to cold injury in the future. All patients who have had frostbite therefore need preventive advice.

Unlike burn injury, frostbite is characterized by mechanisms of injury due to local ischemia as well as direct cellular injury $[2,5]$. Therefore, there is a tendency for delayed healing and even worsening of wounds. In addition, frostbite is also characterized by a very slow course of wound healing. Surgeons should therefore decide on surgery after waiting for a sufficient period of time (3-6 weeks) when surgery is indicated. In addition, it is of ultimate importance to determine the optimal timing of surgery. At our medical institution, we performed surgery after waiting for approximately three weeks until patients achieved a clear demarcation.

Based on our clinical experience and previous reports, we propose the following treatment protocol for patients with frostbite: 1) Surgeons should evaluate the severity of frostbite, the viability of affected tissue, and the perfusion status on imaging modalities in the early stage of onset. In addition, to prevent the possible occurrence of microvascular thrombosis due to the inflammation of the small vascular endothelium and to improve the hemodynamic status, surgeons should use NSAIDs, PGE1, heparin, aspirin, anti-coagulants, growth factors, and hyperbaric oxygen therapy in the initial management of frostbite injury. Then, they should wait for a sufficient period of time until the demarcation of wounds and decide on surgical procedures (skin graft, flap surgery, or amputation) and the optimal timing of 
surgery. 2) After achieving a complete cure, surgeons should perform follow-up with patients for 6-12 months for the prevention of the occurrence of long-term sequelae. In addition, they should encourage patients to wear garments and to perform exercise for the prevention of joint stiffness in the early stage after a complete cure has been achieved. Moreover, they should also educate the patients to avoid re-exposure to cold environments.

Finally, further prospective studies are warranted to examine various approaches to the treatment of frostbite injury, which is essential for establishing a treatment protocol.

\section{REFERENCES}

1. Imray C, Grieve A, Dhillon S, et al. Cold damage to the extremities: frostbite and non-freezing cold injuries. Postgrad Med J 2009;85:481-8.

2. Murphy JV, Banwell PE, Roberts AH, et al. Frostbite: pathogenesis and treatment. J Trauma 2000;48:171-8.

3. Grieve AW, Davis P, Dhillon S, et al. A clinical review of the management of frostbite. J R Army Med Corps 2011;157: 73-8.

4. Mills WJ. Clinical aspects of frostbite injury. In: Eleanor W, editor. Proceedings of the symposium on arctic biology and medicine: IV, frostbite. Fort Wainwright: Arctic Aeromedical Laboratory; 1964.

5. Goertz O, Hirsch T, Buschhaus B, et al. Intravital pathophysiologic comparison of frostbite and burn injury in a murine model. J Surg Res 2011;167:e395-401.
6. Hallam MJ, Cubison T, Dheansa B, et al. Managing frostbite. BMJ 2010;341:c5864.

7. Miller MB, Koltai PJ. Treatment of experimental frostbite with pentoxifylline and aloe vera cream. Arch Otolaryngol Head Neck Surg 1995;121:678-80.

8. McCauley RL, Heggers JP, Robson MC. Frostbite. Methods to minimize tissue loss. Postgrad Med 1990;88:67-8, 73-7.

9. Saito S, Shimada H. Effect of prostaglandin E1 analogue administration on peripheral skin temperature at high altitude. Angiology 1994;45:455-60.

10. Cauchy E, Chetaille E, Marchand V, et al. Retrospective study of 70 cases of severe frostbite lesions: a proposed new classification scheme. Wilderness Environ Med 2001;12: 248-55.

11. Britt LD, Dascombe WH, Rodriguez A. New horizons in management of hypothermia and frostbite injury. Surg Clin North Am 1991;71:345-70.

12. Valnicek SM, Chasmar LR, Clapson JB. Frostbite in the prairies: a 12-year review. Plast Reconstr Surg 1993;92:633-41.

13. Cauchy E, Marsigny B, Allamel G, et al. The value of technetium 99 scintigraphy in the prognosis of amputation in severe frostbite injuries of the extremities: a retrospective study of 92 severe frostbite injuries. J Hand Surg Am 2000; 25:969-78.

14. Barker JR, Haws MJ, Brown RE, et al. Magnetic resonance imaging of severe frostbite injuries. Ann Plast Surg 1997;38: 275-9. 